\title{
Flow Unsteadiness and Pressure Pulsations in a Nuclear Reactor Coolant Pump
}

\author{
Dan Ni* - Minguan Yang - Bo Gao - Ning Zhang - Zhong Li \\ Jiangsu University, School of Energy and Power Engineering, China
}

Unsteady flow induced by rotor-stator interaction is detrimental to the safe operation of the nuclear reactor coolant pump, so it is essential to clarify flow structures and pressure pulsations in such pumps, especially within the spherical casing. In this paper, unsteady flow characteristics in a mixed-flow nuclear reactor coolant model pump were investigated using large-eddy simulation (LES) method. Results show that at the nominal flow rate, in two particular diffuser channels near the spherical casing discharge nozzle, the flow structures are uneven compared with that in the other flow channels. The reason is associated with the position of the flow channel with respect to the spherical casing nozzle. Large- scale flow separation and backflow structures easily occur at the regions near these two channels. In the right and the middle region of the casing nozzle, due to the large-scale separate flow and high vorticity magnitude, unsteady flow structures are more complicated in comparison with the other regions. It has been found that the vorticity spectra and the pressure spectra almost have the same main excitation frequencies. Therefore, it has been confirmed that for particular regions pressure pulsations are determined by the shedding vortex wake from the diffuser blade trailing edge of the nuclear reactor coolant model pump.

Keywords: nuclear reactor coolant pump, flow unsteadiness, pressure pulsation, vorticity magnitude

Highlights

- Numerical investigation of flow unsteadiness and pressure pulsation in a nuclear reactor coolant pump.

- Flow separations and backflows easily occur at the diffuser channels near the discharge nozzle region.

- At the left region of the casing, the discharge nozzle is affected significantly by an intense rotor-stator interaction effect.

- In the right and middle of the casing nozzle, unsteady flow structures are more complicated.

- $\quad$ An unsteady vortex-shedding effect would motivate evident component in pressure spectrum.

\section{O INTRODUCTION}

A nuclear reactor coolant pump (RCP) [1], the device in the reactor core and the steam generator that transfers the heat energy [2], is the "heart" of a nuclear reactor driving the circulation flow of the coolant in the main loop [3]. The nuclear reactor coolant pump is the only rotating part of the nuclear island, so it belongs in a nuclear safety grade one facility. Moreover, it is also the main energy-consuming equipment in the nuclear power plants, which must be guaranteed to operate continuously over a long term and without trouble. An investigation on the internal flow in the mixed-flow nuclear reactor coolant pump is one of the key problems for the development of reactor coolant pumps in large pressurized water reactors. Some investigations have been conducted to study the design of the mixed-flow pump [4] and [5] and flows instabilities [6] and [7]. However, most of them only focus on the unsteady pressure pulsation in impeller [8] and [9] or the performance of the nuclear reactor coolant pump [10] and [11]. The unsteady flow structure of a mixed-flow nuclear reactor coolant pump, especially in certain specific regions, is very important to the safety analysis of a nuclear reactor, as it could generate serious flow-induced vibration, threatening the pump integrity [8]. Therefore, a comprehensive analysis and prediction of pressure pulsation caused by intense rotor-stator interaction are essential for the design of the mixed-flow nuclear reactor coolant pump [12] and [13]. At present, in order to improve the efficiency and stable operation of the nuclear reactor coolant pump, the complicated internal unsteady flow structures should be thoroughly illustrated.

The nuclear reactor coolant pump has a special structure equipped with a spherical casing, which determines a typical and complex flow pattern within the pump. Knierim et al. [14] designed a new type of reactor coolant pump for a $1400 \mathrm{MW}$ nuclear power plant. The impeller and diffuser were gradually optimized based on the computational fluid dynamics (CFD). The volute casing is a spherical shape with a discharge nozzle facing the impeller, and the flow structures in the region around the discharge nozzle are uniform. The region of the discharge nozzle itself is characterized by the fact that the flow below the outlet port is divided into two parts. One portion flows out of the casing discharge nozzle, whereas the other portion circulates around the casing once more prior to exiting. Kato et al. [15] and [16] described internal flows of a high-specific-speed mixed-flow pump at low flow rates using large-eddy simulation (LES), and it showed that the head-flow curve exhibited weak 
instability characteristics. Posa et al. [17] reported the LES method used in a mixed-flow pump, where a structured cylindrical coordinate solver with optimal conservation properties was utilized in conjunction with an immersed-boundary method. Moreover, the overall agreement with the experimental results was excellent, demonstrating the robustness and feasibility of the approach in rotating machinery applications. It demonstrated that the LES method was adequate to predict complicated flow patterns of a high-specificspeed mixed-flow pump. With the development of non-contact measuring techniques, unsteady particle image velocimetry (PIV) [18] and laser Doppler velocimetry (LDV), measuring techniques are often applied to investigate complex unsteady internal flow in pumps, in such a way that no external disturbance is imposed on the flow field. Miyabe et al. [6] and [7] used PIV and pressure fluctuation measurements to investigate the propagation mechanism of a rotating stall in a mixed-flow pump. It was found that unstable performance was caused by periodic large-scale abrupt backflow generated from the diffuser to the impeller outlet. However, most research focuses on the design and the instability flow of the impeller and diffuser in a mixed-flow pump [19] to [22], and unsteady flows in a mixed-flow nuclear RCP with the specific spherical casing are rarely conducted. Consequently, true internal flow structures have not been thoroughly revealed.

In this study, based on the LES method, the internal unsteady flow in a mixed-flow RCP model pump is studied. Monitoring points are mounted on the impeller inlet, diffuser channels, and spherical casing to achieve pressure pulsation characteristics. Finally, pressure pulsations and complicated flow structures are combined together to clarify flow unsteadiness in the RCP model pump.

\section{NUMERICAL METHOD}

\subsection{Governing Equations}

By neglecting the incompressible effect of the fluid, LES governing equations [15] to [17] can be described in the following form.

$$
\begin{gathered}
\frac{\partial}{\partial x_{i}}\left(\rho \bar{u}_{i}\right)=0 \\
\frac{\partial}{\partial t}\left(\rho \bar{u}_{i}\right)+\frac{\partial}{\partial x_{j}}\left(\rho \bar{u}_{i} \bar{u}_{j}\right)=\frac{\partial}{\partial x_{j}}\left(\mu \frac{\partial \sigma_{i j}}{\partial x_{j}}\right)-\frac{\partial \bar{p}}{\partial x_{i}}-\frac{\partial \tau_{i j}}{\partial x_{j}},
\end{gathered}
$$

where $\bar{u}_{i} \quad(i=1,2,3)$ is the grid-scale velocity component, $\bar{p}$ is the grid-scale static pressure, $\rho$ is the density and $\mu$ is the kinematic viscosity, and $\sigma_{i j}$ is the stress tensor having the form of Eq. (3).

$$
\sigma_{i j}=\left[\mu\left(\frac{\partial \bar{u}_{i}}{\partial x_{j}}+\frac{\partial \bar{u}_{j}}{\partial x_{i}}\right)\right]-\frac{2}{3} \mu \frac{\partial \bar{u}_{i}}{\partial x_{i}} \delta_{i j},
$$

where $\delta_{i j}$ is Kronecker delta. $\tau_{i j}$ is the sub-grid-scale (SGS) stress tensor, which is defined in Eq.(4).

$$
\tau_{i j}=\rho \overline{u_{i} u_{j}}-\rho \bar{u}_{i} \bar{u}_{j} \text {. }
$$

In this study, the Smagorinsky-Lilly SGS fixedcoefficient model [23] is applied to close the equations. In this model, the eddy viscosity is defined in Eq. (5).

$$
\mu_{t}=\rho L_{s}^{2}|\bar{S}|,
$$

where $L_{S}$ is the mixed length of the grid and $|\bar{S}|=\sqrt{2 \bar{S}_{i j} \bar{S}_{i j}}$, where $S_{i j}$ is the deformation tensor. The function of $L_{S}$ is defined in Eq. (6).

$$
L_{s}=\min \left(k d, C_{s} V^{1 / 3}\right),
$$

where $k$ is the von-Kármán constant, $d$ is the distance to the nearest wall, $V$ is the volume of the computational cells and $C_{s}$ is the Smagorinsky constant. $C_{S}$ is usually a constant 0.1 .

\subsection{Mesh Generation}

A mixed-flow nuclear reactor coolant model pump was first designed. The main parameters of the RCP model pump are shown in Table 1. The RCP mainly consists of an impeller, diffuser, and spherical casing. The structure of the RCP model pump is illustrated in Fig. 1.

The quality of the mesh has a critical influence on the numerical simulation accuracy. The AnsysICEM mesh generation tool was adopted to generate structured mesh cells of the model pump in order to obtain precise unsteady flow structures and pressure fluctuation characteristics. To guarantee the computational accuracy, the fine grids were necessarily required by the LES model. Thus, grid cells near the solid wall were encrypted, especially on the surface of the blades, where significant pressure gradients and flow separation may easily occur [12] and [13]. Meanwhile, a detailed grid sensitivity analysis with four different mesh numbers has been carried out, as summarized in Table 2. After mesh sensitivity checks, it is noted that the pump head under nominal flow rate decrease $0.2 \%$ with the mesh number increasing and, finally, the value of the pump head is constant. Finally, Case 3 of a total mesh number nearly $7 \times 10^{6}$ is selected for numerical simulation considering the 
current computational ability. The numerical results demonstrate that at the near wall region of the impeller and the diffuser, the $y+$ value is about 1 . In the other region of the impeller and the diffuser, the $y+$ value is lower than 5. Finally, the averaged $y+$ value of the whole computational domain of the impeller and the diffuser is approximately 4.5 , which could provide adequate resolution in the critical regions of the computation domain. Fig. 2 shows the mesh of the RCP model pump impeller.

Table 1. Main design parameters

\begin{tabular}{lc}
\hline Parameters & Values \\
\hline Nominal flow rate $Q_{N}$ & $848 \mathrm{~m}^{3} / \mathrm{h}=0.236 \mathrm{~m}^{3} / \mathrm{s}$ \\
\hline Designed head $H_{N}$ & $12.7 \mathrm{~m}$ \\
\hline Rotating speed $n$ & $1480 \mathrm{r} / \mathrm{min}$ \\
\hline Specific speed $n_{s}=\left(3.65 n \sqrt{Q_{N}}\right) / H_{N}{ }^{0.75}$ & 390 \\
\hline Impeller outlet diameter $d_{2}$ & $268 \mathrm{~mm}$ \\
\hline Impeller outlet width $b_{2}$ & $84 \mathrm{~mm}$ \\
\hline Casing diameter $D$ & $637.5 \mathrm{~mm}$ \\
\hline Impeller blade number $Z_{i}$ & 4 \\
\hline Diffuser blade number $Z_{d}$ & 12 \\
\hline
\end{tabular}

Table 2. Mesh sensitivity check

\begin{tabular}{lrrrr}
\hline Parameters & \multicolumn{1}{c}{ Case 1 } & \multicolumn{1}{c}{ Case 2 } & \multicolumn{1}{c}{ Case 3 } & \multicolumn{1}{c}{ Case 4 } \\
\hline Inlet section & 136,567 & 257,863 & 336,835 & 461,823 \\
\hline Impeller & $1,237,651$ & $2,210,789$ & $3,231,400$ & $4,247,913$ \\
\hline Diffuser & 884,132 & $1,007,541$ & $1,230,624$ & $1,545,004$ \\
\hline Spherical casing & $1,060,434$ & $1,735,866$ & $2,137,419$ & $3,845,103$ \\
\hline Outlet section & 184,471 & 360,547 & 502,281 & 651,079 \\
\hline Total elements & $3,503,255$ & $5,572,606$ & $7,438,559$ & $10,750,922$ \\
\hline $\begin{array}{l}\text { Pump head in } \\
\text { design point [m] }\end{array}$ & 12.405 & 12.378 & 12.369 & 12.365 \\
\hline
\end{tabular}

\subsection{Solution Parameters}

The LES method [26] and [27] has a better ability to predict unsteady flow than the RANS method in general [28] and [29], especially for the flow separation [30] to [32]. Before the calculation of transient LES simulation, the steady calculation should be achieved. In this study, steady calculation using the standard $k-\varepsilon$ model is first conducted, and the results are set as the initial boundary for transient LES simulation. The pump inlet is defined as the velocity inlet boundary condition and a constant pressure $p=101325 \mathrm{~Pa}$ is imposed at the pump outlet. The coupling of the rotational domain (impeller) and the stationary domain (diffuser) is solved by moving mesh.

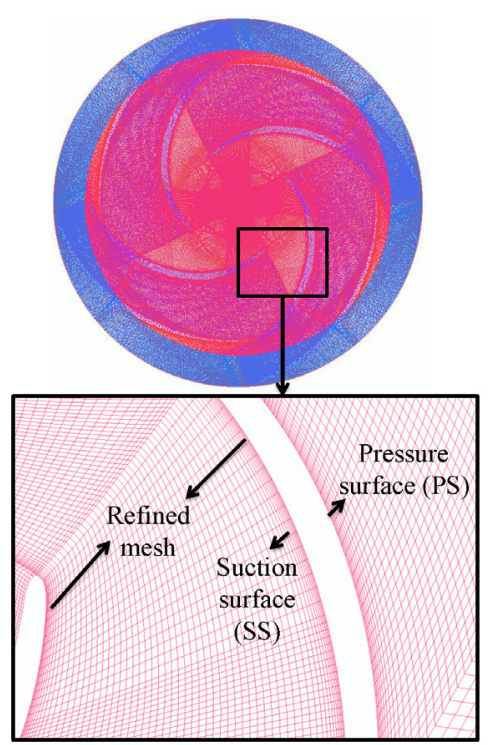

Fig. 2. The mesh of the impeller and its partially refined structured grids

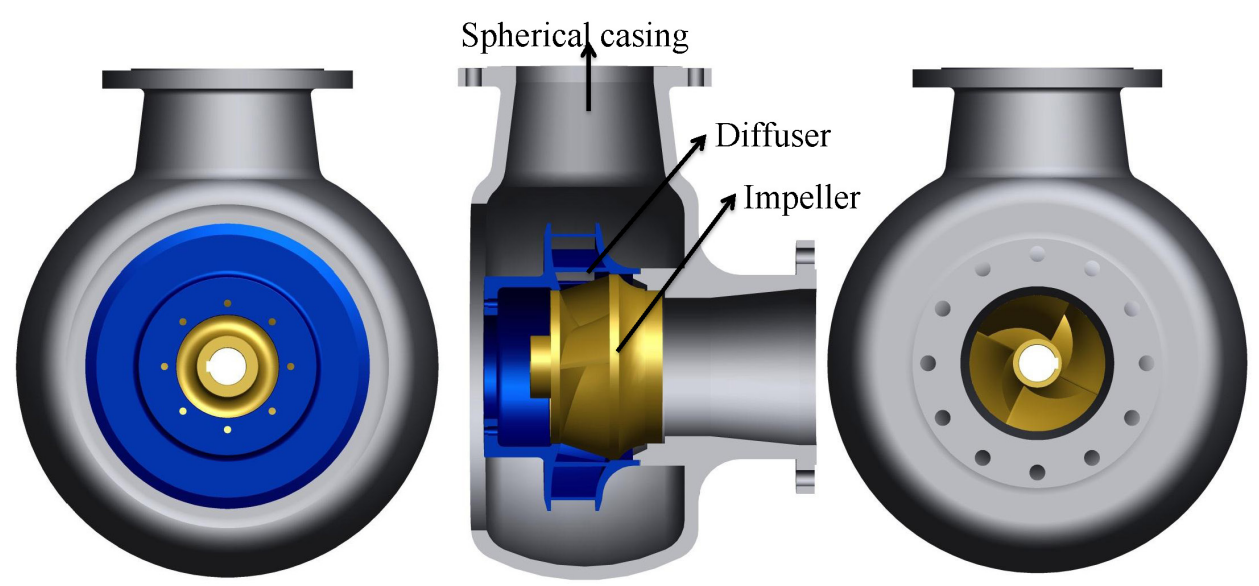

Fig. 1. Diagram of the mixed-flow RCP model pump 
In order to illustrate the flow unsteadiness characteristics of the RCP model pump, especially within the spherical casing, time step size is set as $\Delta t=112.6 \mu \mathrm{s}$ in order to have enough resolution of unsteady flow results during calculation. Thus, each impeller revolution will be calculated in a time sequence of 360 time steps corresponding to $1^{\circ}$ of the impeller rotation speed. The numerical residual convergence criterion is set as $10^{-5}$ in order to ensure the result to be converged. The unsteady convergence also has a great influence on the achieved results, so, at least, fifty revolutions were achieved to guarantee the numerical accuracy.

a)

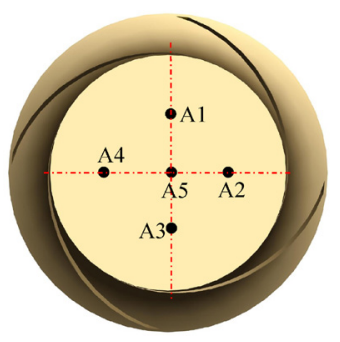

b)

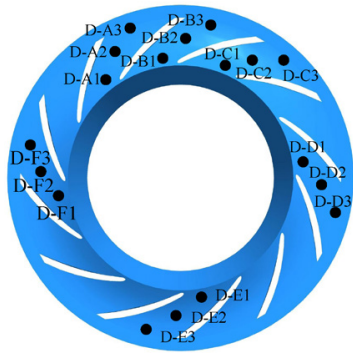

c)

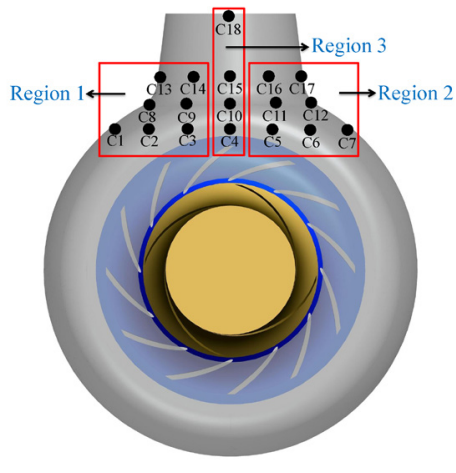

Fig. 3. Details of the monitoring points on the RCP model pump; a) impeller inlet, b) diffuser channels, and c) discharge nozzle of spherical casing

\subsection{Monitoring Points}

Monitoring points are mounted on the principal districts to obtain the comprehensive understanding of flow unsteadiness characteristics. Many researchers have already investigated the unsteady characteristics within impeller channels [9], so the emphasis is laid upon pressure pulsation within the diffuser channels, the spherical casing, and the pump inlet. Therefore, five monitoring points are mounted on the impeller inlet as seen in Fig. 3a. Fig. 3b shows monitoring points in six diffuser channels out of a total of 12 flow channels. All the points are locating at the midspan plane of the diffuser. The diffuser channel on three monitoring points (such as D-A1, D-A2, and
D-A3) is defined as Channel A. The Channel B and Channel $\mathrm{C}$ have similar definitions. It is well known that unsteady flow distribution in the spherical casing of the RCP is quite complicated, especially at the area near the discharge nozzle of casing [14]. Thus, monitoring points are mounted on the mid-span plane of the spherical casing nearing the discharge nozzle zone as shown in Fig. 3c. As discussed in the preceding, the complicated flow pattern is expected in this region. Therefore, a total of 18 monitoring points are selected for investigation in this region. These points are divided into three groups for later analysis as shown in Fig. 3c.

\section{EXPERIMENTAL SETUP}

In order to validate the accuracy of the current numerical method, experimental investigation of the RCP model pump is carried out in a closed-loop test rig to guarantee the measuring accuracy. Details of the test loop are shown in Fig. 4. The water temperature is about $25{ }^{\circ} \mathrm{C}$ during experiments. Flow rates of the RCP at various conditions are measured with an electronic flow meter with an absolute accuracy of

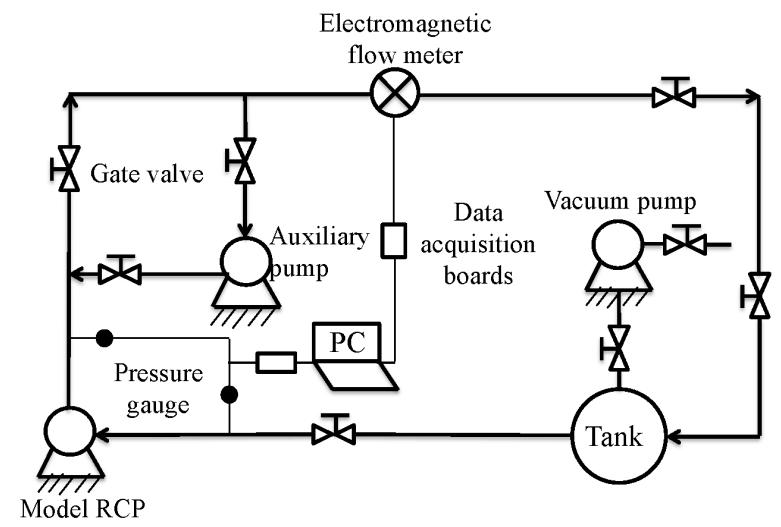

Fig. 4. Closed test loop

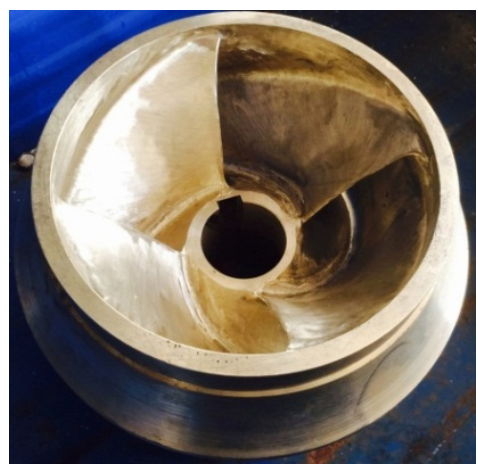

Fig. 5. Test used impeller 
$\pm 0.2 \%$ of the measured value. Two pressure gauges at the pump inlet and outlet pipings are used to obtain the pump head with an accuracy of $\pm 1 \%$. An auxiliary pump is used to overcome the pressure drop in the system. The rotating speed of the RCP model pump was ensured to be at the design value $1480 \mathrm{r} / \mathrm{min}$ by adopting a frequency inverter during the experimental process. Fig. 5 depicts the test of the used impeller.

\section{RESULTS AND DISCUSSIONS}

\subsection{Validation of the Numerical Method}

In order to validate the reliability of CFD analysis, CFD results are compared with experimental results of the RCP model pump. Fig. 6 shows the comparisons between experiments and the numerical results obtained from transient LES models. As observed, the maximum difference from the comparison is about $3.5 \%$. At nominal flow rates, the difference between the predicted and measured values is about $2.4 \%$. The discrepancy between CFD and experimental results may be attributed to the grid resolution around the blade surface, and the current mesh resolution may be insufficient to resolve the turbulent boundary layer developing on the blade surface. Furthermore, effects of the leakage flow through the clearance are not considered in the present numerical simulation [15]. However, it can be found that the tendency of CFD results has a good agreement with the test results. From performance comparison, it can be concluded that the current numerical method could capture the mainly unsteady flow structures of the RCP model pump.

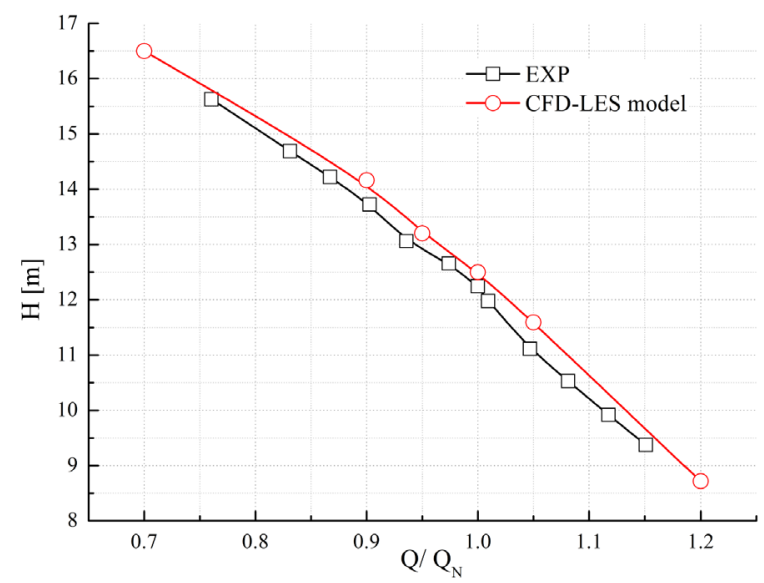

Fig. 6. Performance comparison between experimental and numerical results

\subsection{Unsteady Pressure Pulsation Characteristics at the Impeller Inlet}

In order to analyse the unsteady pressure pulsations, pressure coefficient $C_{p}$ is defined in Eq. (7).

$$
\begin{gathered}
C_{p}=\Delta P /\left(0.5 \rho u_{2}^{2}\right), \\
u_{2}=\frac{n \pi D_{2}}{60}, \\
\Delta C_{p}=C_{p \max }-C_{p \min },
\end{gathered}
$$

where $\Delta P$ is pressure amplitude, $u_{2}$ is tangential velocity at the impeller outlet, and $D_{2}$ is the impeller outlet diameter. $\Delta C_{p}$ is the difference value, which is the maximum of $C_{p}$ minus minimum of $C_{p}$.

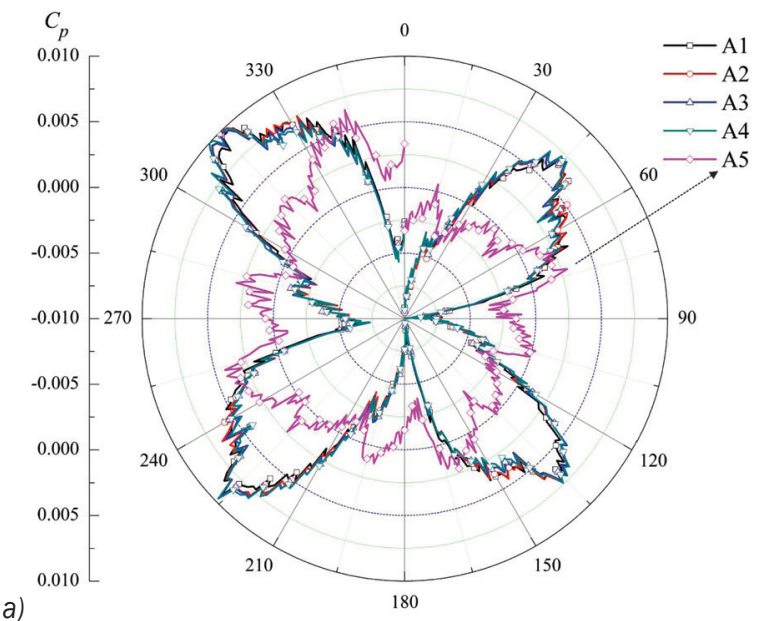

a)

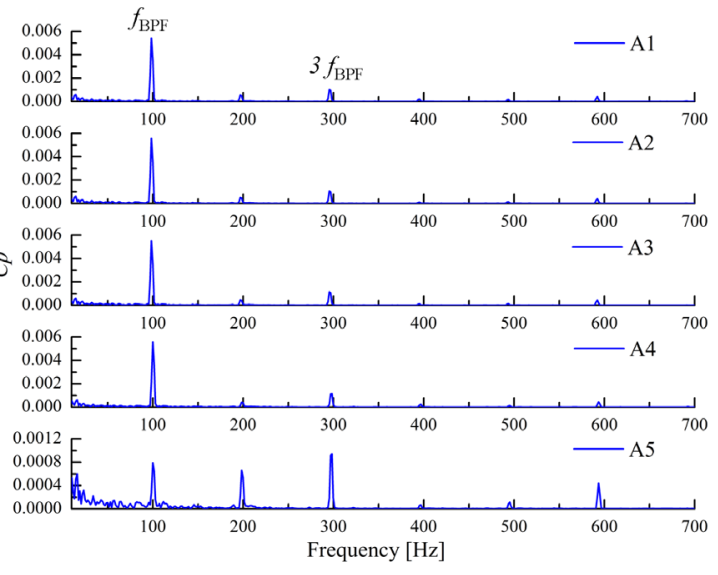

Fig. 7. a) Pressure coefficients of the monitoring points at the impeller inlet, and b) frequency domain of each monitoring point at impeller inlet

The rotating speed of the impeller keeps a constant value of $1480 \mathrm{r} / \mathrm{min}$, so the impeller-rotating frequency $\left(f_{R}\right)$ is $24.7 \mathrm{~Hz}$ and the blade-passing frequency 
$\left(f_{\mathrm{BPF}}\right)$ is $99 \mathrm{~Hz}$. Fig. 7a shows pressure coefficients of the monitoring points in the impeller inlet during one impeller-rotating period. It is observed that the pressure signal of the points fluctuates significantly with the impeller rotating, and pressure coefficients of the monitoring points (A1 A4) have an approximately symmetrical distribution in one impeller rotating cycle. Differences of pressure coefficient among these four monitoring points are quite small. It indicates that pressure signals in the periphery of the impeller inlet are nearly consistent with the impeller rotating. However, the point A5 at impeller inlet centre shows different pressure coefficient distribution compared with the other four measuring points, and the pressure coefficient value is lower than others. It does not show any obvious periodicity in one rotating cycle. The fast Fourier transform (FFT) method was adopted to change the time domain signals into the frequency domain signals to analyse pressure spectra. The hanning window was adopted to reduce the energy leakage during signal process [33]. Fig. $7 \mathrm{~b}$ shows pressure spectra of the monitoring points at the impeller inlet. The circumferential monitoring points (A1 A4) have similar pressure spectra, and the dominant peaks occur at the blade-passing frequency $\left(f_{\mathrm{BPF}}\right)$. Moreover, high harmonics at $297 \mathrm{~Hz}\left(3 f_{\mathrm{BPF}}\right)$ could also be identified with an amplitude about $1 / 6$ of that at $f_{\mathrm{BPF}}$. However, the predominant component of the point in the impeller inlet centre (A5) occurs at three times the blade-passing frequency $\left(3 f_{\mathrm{BPF}}\right)$.

\subsection{Unsteady Pressure Pulsation Characteristics within the Diffuser Channels}

Fig. 8 presents pressure coefficient of monitoring points within three diffuser channels near the discharge nozzle in one rotating cycle. It is evident that pressure signals change periodically, and four crests and troughs exist during one impeller revolution due to rotor-stator interaction. From the diffuser inlet to the outlet, when the pressure coefficients present wave crests, the pressure amplitudes gradually decrease and when they present wave troughs, the amplitude increases gradually in one rotating cycle. Obviously, near the diffuser channel inlet, the pressure coefficients fluctuate more greatly than the diffuser outlet. It is indicated that rotor-stator interaction effect is obvious, and it is weakened gradually along with the diffuser channels. Meanwhile, pressure coefficients of these three diffuser channels have different distributions.

In order to quantitatively analyse this phenomenon, the difference values of pressure

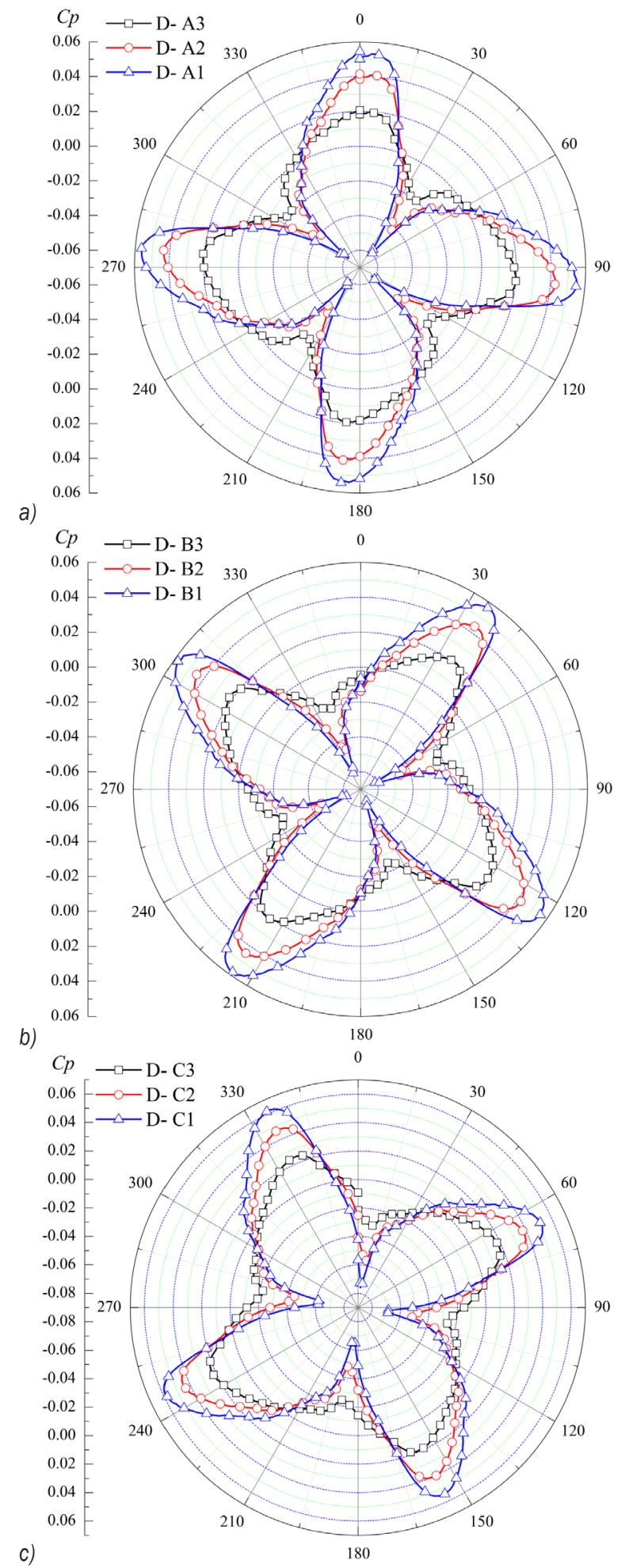

Fig. 8. Pressure coefficient in diffuser: a) Channel A, b) Channel B, and c) Channel $\mathrm{C}$

coefficients are analysed on the diffuser monitoring points in a rotating cycle, as shown in Fig. 9. It is 
indicated that diffusers Channel $\mathrm{C}$ and Channel B have relatively large difference values of the pressure coefficient, especially for Channel C. These two flow channels are near the casing discharge nozzle. Thus, it is concluded that in a rotating cycle in these two flow channels, the flow structure is a more uneven compared with other flow channels. The reason is associated with the location of the flow channel, and larger flow separations or backflows occur easily near the two flow channels.

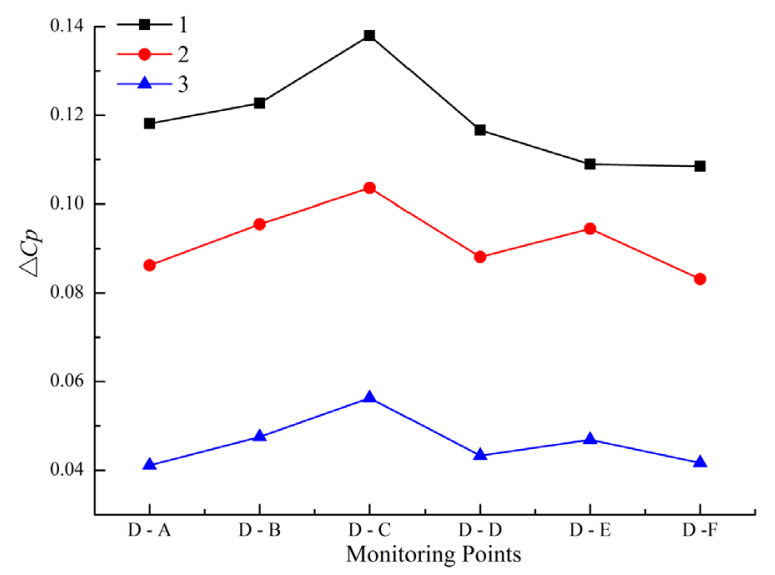

Fig. 9. Pressure coefficients difference values on diffuser monitoring point
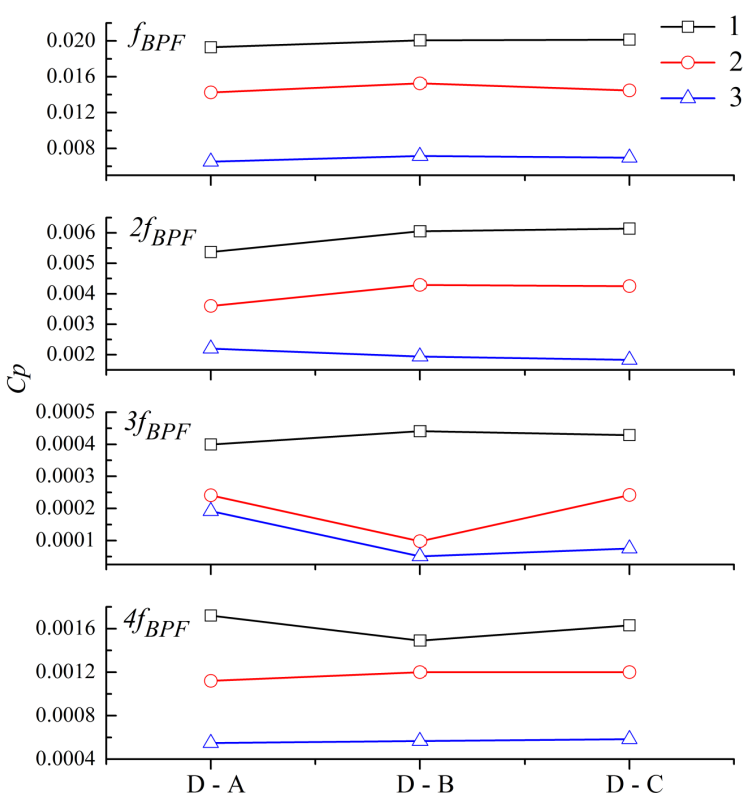

Fig. 10. Pressure pulsation amplitudes at blade-passing frequency $\left(f_{\mathrm{BPF}}\right)$ and higher harmonic

Fig. 10 shows the pressure pulsation amplitude at the blade-passing frequency $\left(f_{\mathrm{BPF}}\right)$ and its high harmonic of monitoring points in diffuser channels
(Channels A C). Obviously, along with the flow channels, the blade-passing frequency and higher harmonic become increasingly weak, and the main excitation frequency in the diffuser channel is the blade-passing frequency $\left(f_{\mathrm{BPF}}\right)$. Pressure pulsation amplitude values at the blade-passing frequency $\left(f_{\mathrm{BPF}}\right)$ and its second harmonic $\left(2 f_{\mathrm{BPF}}\right)$ in Channel $\mathrm{B}$ are larger than the other two channels. Channel $\mathrm{C}$ has the second largest amplitude value, and the pressure pulsation amplitude value is minimum in Channel A. However, at higher harmonics $\left(3 f_{\mathrm{BPF}}\right.$ and $4 f_{\mathrm{BPF}}$ ), there are some different features, especially in Channel B. At $3 f_{\mathrm{BPF}}$, the amplitude values of Point D-B3 and Point D-B2 in diffuser Channel B are lower than the other channels. Meanwhile, at 4 $f_{\mathrm{BPF}}$, the amplitude of Point D-B1 is less than Point $\mathrm{D}-\mathrm{A} 1$ and Point $\mathrm{D}-\mathrm{C} 1$. That is because some other complex unsteadiness flows in flow Channel B and the rotor-stator interaction effects become weaker. Thus, it is indicated that the blade-passing frequency due to the rotor-stator interaction effect is obviously different in every diffuser channel. Furthermore, internal flows in different diffuser channels, especially near the spherical casing discharge nozzle region, are complicated.

\subsection{Unsteady Pressure Pulsation Characteristics within Spherical Casing}

Due to the particular spherical casing, flow structures show a greater difference in comparison with the conventional spiral volute. From this perspective, it is important to analyse unsteady flow characteristics within the spherical casing.

In order to analyse the unsteady pressure spectra in the spherical casing, Figs. 11 to 13 show the pressure pulsation characteristics around the discharge nozzle region. Monitoring points at the discharge nozzle region are divided into three groups symmetrically. Region 1 is located on the left of the discharge nozzle. Region 2 is located in the right of the discharge nozzle, and Region 3 is located in the middle of the discharge nozzle. The vertical scales in Figs. 11 to 13 are different, due to the increasing role of the low frequencies components.

Fig. 11 presents the pressure spectra in Region 1. From the left side of the casing to the middle of the casing, pressure amplitudes at the blade-passing frequency $\left(f_{\mathrm{BPF}}\right)$ and its high harmonic $\left(2 f_{\mathrm{BPF}}\right)$ decrease gradually. Near the wall surface, these monitoring points $(\mathrm{C} 1, \mathrm{C} 2, \mathrm{C} 8$, and $\mathrm{C} 13)$ have the main excitation frequency, which is the blade-passing frequency $\left(f_{\mathrm{BPF}}\right)$. It is indicated that the rotor-stator interaction effect is 

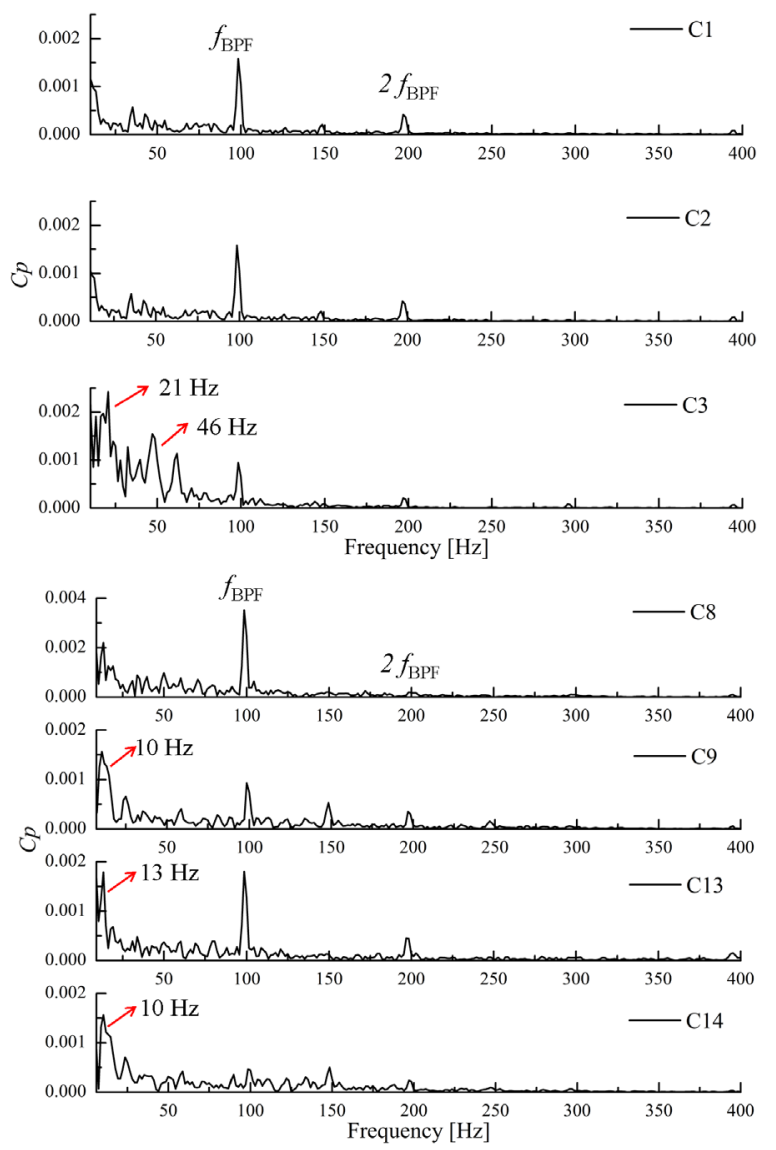

Fig. 11. Pressure spectra of monitoring points in Region 1

significant. However, the monitoring point $\mathrm{C} 3$ has a complex pressure spectrum as indicated by many peaks appearing. As observed, the dominant excitation frequency is $21 \mathrm{~Hz}$, and the second larger peak is 46 $\mathrm{Hz}$. The interval of frequencies is about $20 \mathrm{~Hz}$ to 50 $\mathrm{Hz}$ in the low-frequency band. These low-frequency signals are related to the complicated internal flow structure developing at the casing discharge nozzle region. For $\mathrm{C} 9, \mathrm{C} 13$, and $\mathrm{C} 14$, significant excitation frequencies occur within the low-frequency band, i.e. $10 \mathrm{~Hz}, 13 \mathrm{~Hz}$, and $10 \mathrm{~Hz}$ respectively. The interval of the main frequencies is approximately $10 \mathrm{~Hz}$ to $15 \mathrm{~Hz}$. Therefore, from analysis of the pressure spectra characteristics in Region 1, it is concluded that pressure pulsations of the points at the left of the casing discharge nozzle are affected significantly by an intense rotor-stator interaction effect. However, for the points near the middle of the casing discharge nozzle, the rotor-stator interaction effect is less significant, and the dominant component mainly appears in the low-frequency band.
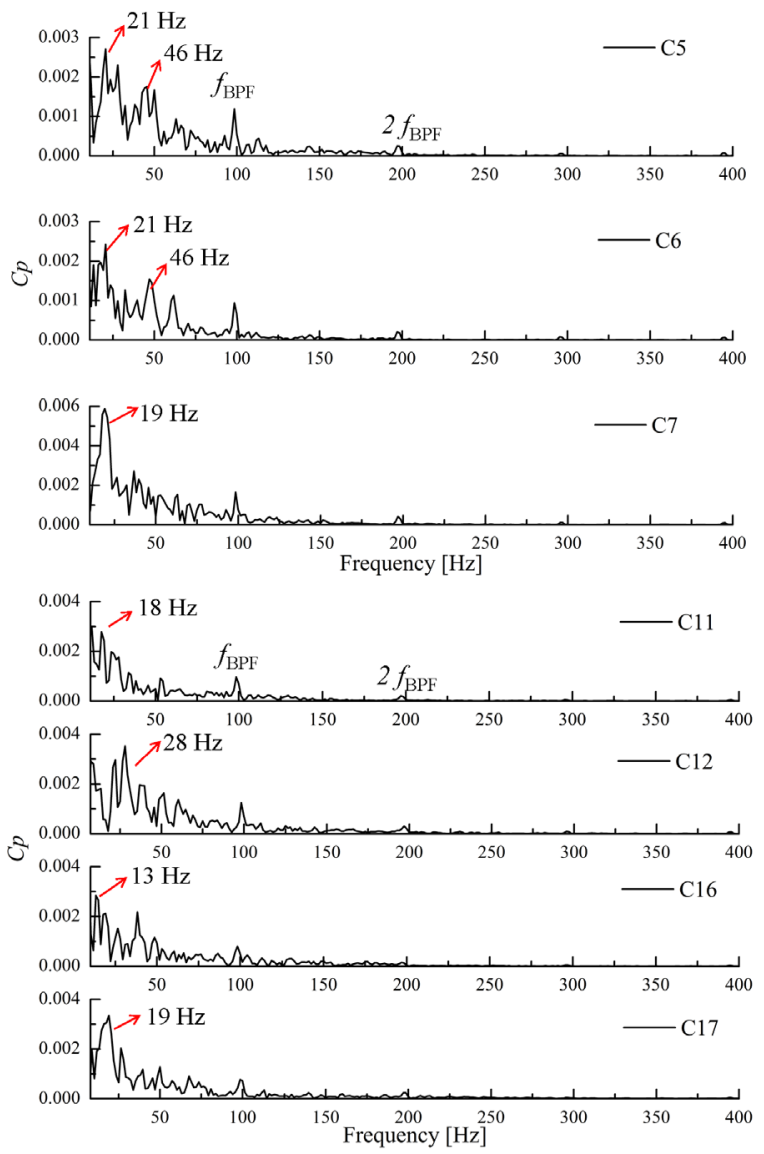

Fig. 12. Pressure spectra of monitoring points in Region 2

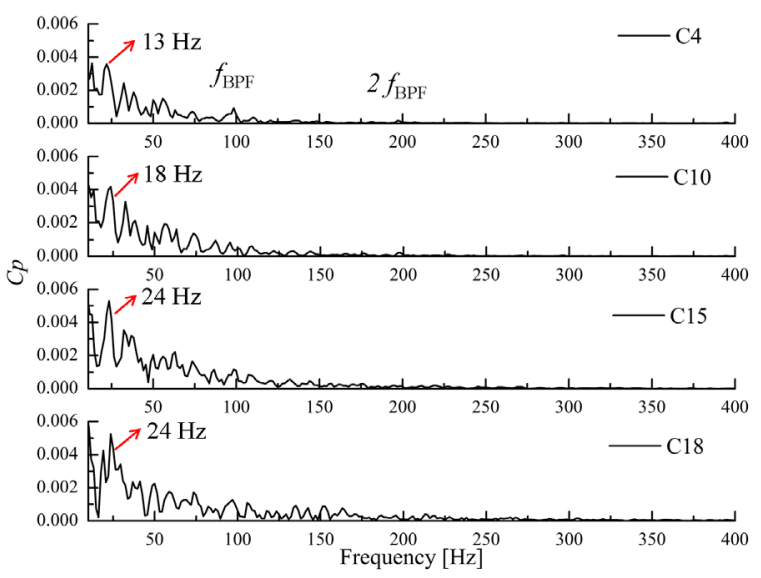

Fig. 13. Pressure spectra of monitoring points in Region 3

Pressure spectra of monitoring points in Region 2 are presented in Fig. 12. It is noted that pressure spectrum characteristics of the points in Region 2 are more complex compared with that in Region 1. Components at the blade-passing frequency and its second harmonic are weaker. As observed, the main 
excitation frequencies of different monitoring points differ obviously, namely $21 \mathrm{~Hz}$ for C5 and C6, $19 \mathrm{~Hz}$ for $\mathrm{C} 7$ and $\mathrm{C} 17,18 \mathrm{~Hz}$ for $\mathrm{C} 11,28 \mathrm{~Hz}$ for $\mathrm{C} 12$ and $13 \mathrm{~Hz}$ for $\mathrm{C} 16$. The interval of the main frequencies can be defined between $10 \mathrm{~Hz}$ and $50 \mathrm{~Hz}$. Therefore, it can be concluded from pressure spectrum analysis, for the monitoring points at the right region of the casing discharge nozzle, that the rotor-stator interaction is not the dominant source of pressure fluctuations. In this region, many components in the low-frequency band are captured, which are related to the complex flow structures developing in this area.

Fig. 13 presents pressure spectra of the monitoring points in Region 3. As noted, components at the blade-passing frequency almost could not be observed, so the rotor-stator interaction effect in this region is also not significant. In the low-frequency band, the main excitation frequency of the monitoring point of $\mathrm{C} 4$ is $13 \mathrm{~Hz}$. For C10, evident peaks occur at $18 \mathrm{~Hz}$ and $24 \mathrm{~Hz}$ for $\mathrm{C} 15, \mathrm{C} 18$. In this region, the interval of the main frequencies is $10 \mathrm{~Hz}$ to $50 \mathrm{~Hz}$.

Table 3. Main excitation frequency of each monitoring point

\begin{tabular}{ccc}
\hline Monitoring points & $\begin{array}{c}\text { Main excitation } \\
\text { frequency [Hz] }\end{array}$ & $\begin{array}{c}\text { Main frequencies } \\
\text { interval [Hz] }\end{array}$ \\
\hline C1, C2, C8, C13 & $f_{\mathrm{BPF}}=99$ & $f_{\mathrm{BPF}}$ \\
\hline $\mathrm{C} 3, \mathrm{C} 5, \mathrm{C} 6$ & 21,46 & 20 to 50 \\
\hline $\mathrm{C} 10, \mathrm{C} 11$ & 18 & 10 to 20 \\
\hline $\mathrm{C} 9, \mathrm{C} 14$ & 10 & 10 to 15 \\
\hline $\mathrm{C} 15, \mathrm{C} 18$ & 24 & 20 to 50 \\
\hline $\mathrm{C} 7, \mathrm{C} 17$ & 19 & 10 to 20 \\
\hline $\mathrm{C} 4, \mathrm{C} 16$ & 13 & 10 to 20 \\
\hline $\mathrm{C} 12$ & 28 & 20 to 50 \\
\hline
\end{tabular}

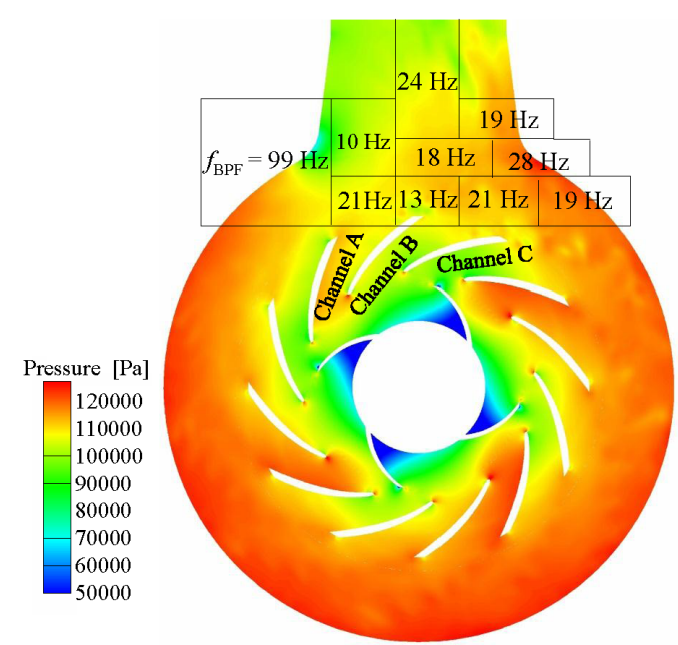

Fig. 14. Distributions of static pressure at the centre plane marked with the main excitation frequencies
The main excitation frequency of each monitoring point is concluded in Table 3. Fig. 14 gives the details of the static pressure distribution at the centre plane of the casing. From Table 3 and Fig. 14 , it can be concluded that the pressure spectra of the points near the left of the casing nozzle are dominated by rotor-stator interaction. And in this region, flow separation does not occur. However, near the middle and right of the spherical casing discharge nozzle, several dominant peaks of low frequencies occur in pressure spectra. Although these frequencies are very complicated, there are some connections between these low frequencies. Near the outlet of the diffuser Channels A and B, the same frequency at about $21 \mathrm{~Hz}$ occurs. It is associated with complex flow structures at this region, including the vortex shedding from the diffuser blade exit and even backflow near the outlet of Channels A and B. However, for the points at the diffuser blade outlet between Channels B and $\mathrm{C}$, a peak at $13 \mathrm{~Hz}$ dominates the pressure spectrum. This excitation frequency is caused by the shedding wake effect from the blade outlet. Near the right side of the spherical casing discharge nozzle, some different frequencies are found. Because of the highvelocity flow, unsteadiness fluid from Channels B and $\mathrm{C}$ impinges on the wall, and some complicated flow structures including large-scale flow separation and backflow are generated in this region, causing the main excitation frequencies.

From the above analysis, it is inferred that unsteady flow structures in diffuser channels and spherical casing discharge nozzle have a huge impact on pressure pulsations of the RCP model pump. Fig. 15 shows typical distributions of velocity for one moment at the centre plane of the casing. It is found that two typical backflows occur in Region 2. One can be defined as the run-through flow, which is located at the diffuser Channel B outlet, and it flows out of the casing discharge nozzle. And the other backflow can be defined as the circulating flow, which is located at the diffuser Channel $\mathrm{C}$ outlet, and it flows around the casing once more prior to exiting. Fig. 16 gives the typical instantaneous distributions of vorticity magnitude at the centre plane of the RCP. It can be clearly seen that a strong vorticity magnitude region near the diffuser Blade $\mathrm{B}$ and diffuser Blade $\mathrm{C}$ occur within the casing. Thus, in Region 2, due to the largescale flow separation (the run-through flow and the circulating flow) and high vorticity magnitude, unsteady flow structures are more complicated than that in the other region.

In order to find the reason behind the complicated pressure pulsations in Region 2, Fig. 17 gives the 


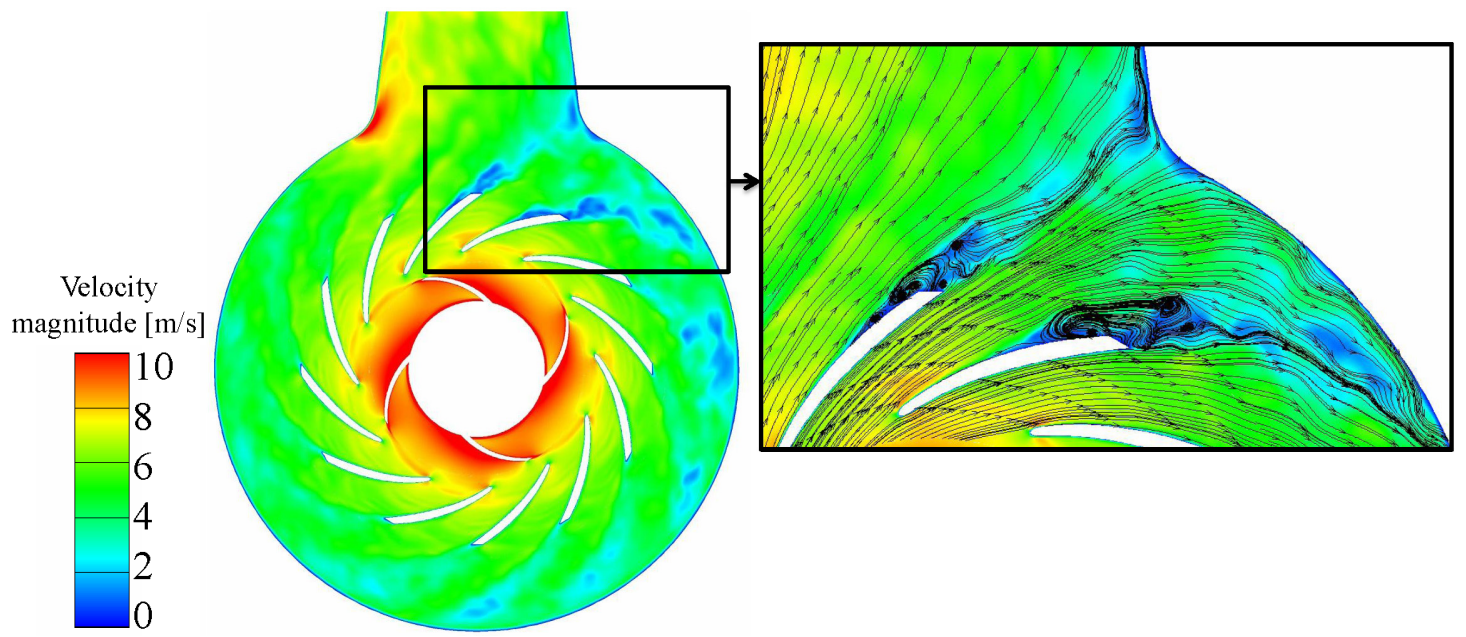

Fig. 15. Typical velocity distribution at the centre plane

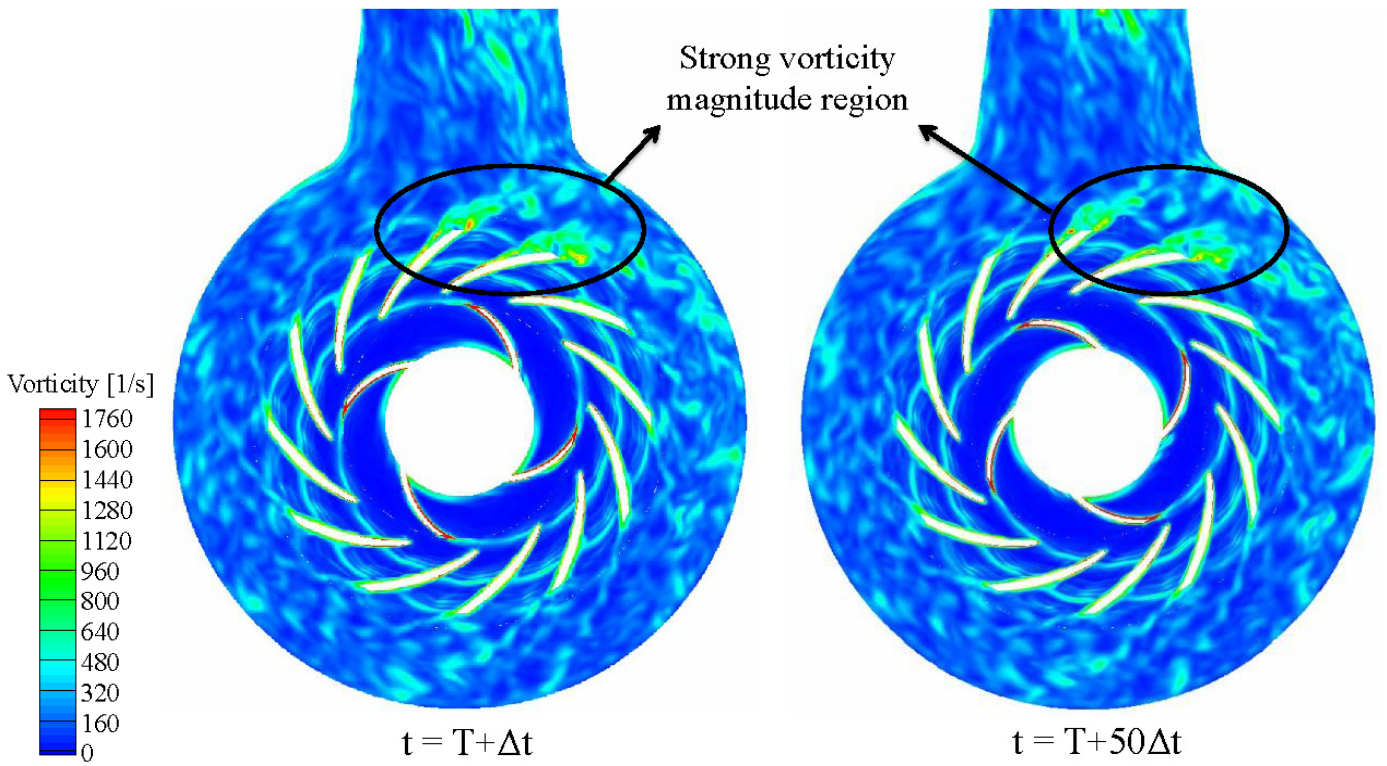

Fig. 16. Typical instantaneous distributions of vorticity magnitude at the mid-span plane of the casing

vorticity spectra of some monitoring points. Combined with Figs. 11 to 13 and Table 3, it is found that the vorticity magnitude and pressure coefficient have the same main excitation: about $13 \mathrm{~Hz}$ for $\mathrm{C} 4,21 \mathrm{~Hz}$ and $46 \mathrm{~Hz}$ for $\mathrm{C} 5,46 \mathrm{~Hz}$ for $\mathrm{C} 6$. Thus, it indicates that an unsteady vortex shedding effect would motivate evident component in pressure spectrum. In summary, in the right and middle of the casing discharge nozzle (Region 2 and Region 3), the internal flow structures are more complicated, as manifested by the large-scale flow separation, the backflow and the high magnitude vortex shedding from the diffuser exit in comparison with that in Region 1.

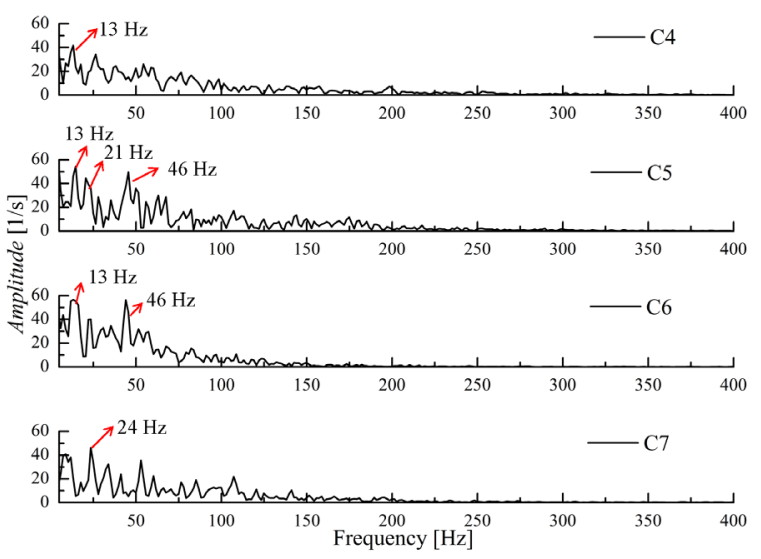

Fig. 17. Vorticity spectra of particular monitoring points 


\section{CONCLUSIONS}

Based on the LES method, the internal unsteady flow in a mixed-flow nuclear RCP at the rated condition is studied in this paper. Some conclusions are made.

Due to the particularity of the spherical casing in the RCP, the internal flow structure is especially ambiguous and complicated. Research results show that at the nominal flow rate, flow structures differ significantly in different diffuser channels, and it is closely associated with the position of the diffuser channel related to the casing nozzle. At the diffuser channels near the nozzle region, flow separations and backflows easily occur.

At the left region of the casing discharge nozzle is affected significantly by an intense rotor-stator interaction effect. However, in the right and middle of the casing nozzle, due to the large-scale separated flow (the run-through flow and the circulating flow) and high vorticity magnitude, unsteady flow structures are more complicated in comparison with the other regions. Moreover, vorticity and pressure spectra almost have the same main excitation frequency. It is believed that some peaks in pressure spectra are caused by the shedding vortex wake from the diffuser blade outlet for some specific regions.

In further study, the investigation would be concentrated on the relationship between the unsteady flow structures and pressure pulsation characteristics using PIV and pressure pulsation measuring techniques in the mixed-flow nuclear reactor coolant model pump for all the concerned conditions.

\section{ACKNOWLEDGMENT}

The authors gratefully acknowledge the financial support of National Natural Science Foundation of China (51476070, 51206063, 51576090), a Project Funded by the Priority Academic Program Development of Jiangsu Higher Education Institutions (PAPD).

\section{REFERENCES}

[1] Gao, H., Gao, F., Zhao, X., Chen, J., Cao, X. (2011). Transient flow analysis in reactor coolant pump systems during flow coastdown period. Nuclear Engineering and Design, vol. 241, no. 2, p. 509-514, D0l:10.1016/j.nucengdes.2010.09.033.

[2] Poullikkas, A. (2000). Two phase flow performance of nuclear reactor cooling pumps. Progress in Nuclear Energy, vol. 36, no. 2, p. 123-130, DOI:10.1016/S0149-1970(00)00007-X.

[3] Cho, Y.-J., Kim, Y.-S., Cho, S., Kim, S., Bae, B.-U., Chung, H.-J., Youn, Y.-J., Park, J.-K., Choi, H.-S., Jeon, W.-J. Kim, B.-D., Kwon, T.-S., Song, C.-H. (2014). Advancement of reactor coolant pump
(RCP) performance verification test in KAERI. Proceedings of the $22^{\text {nd }}$ International Conference on Nuclear Engineering, vol. 2B, p. 1-6, D0l:10.1016/S0149-1970(00)00007-X.

[4] Bing, H., Cao, S.L. (2013). Multi-parameter optimization design, numerical simulation and performance test of mixedflow pump impeller. Science China Technological Sciences, vol. 56, no. 9, p. 2194-2206, Dol:10.1007/s11431-013-53080.

[5] Kim, S., Lee, K.-Y., Kim, J.-H., Kim, J.-H., Jung, U.-H., Choi, Y.S. (2015). High performance hydraulic design techniques of mixed-flow pump impeller and diffuser. Journal of Mechanical Science and Technology, vol. 29, no. 1, p. 227-240, DOI:10.1007/s12206-014-1229-5.

[6] Miyabe, M., Furukawa, A., Maeda, H., Umeki, I., Jittani, Y. (2008). On improvement of characteristic instability and internal flow in mixed flow pumps. Journal of Fluid Science and Technology, vol. 3, no. 6, p. 732-743, D0l:10.1299/jfst.3.732.

[7] Miyabe, M., Maeda, H., Umeki, I., Jittani, Y., (2006). Unstable head-flow characteristic generation mechanism of a low specific speed mixed flow pump. Journal of Thermal Science, vol. 15, no. 2, p. 115-120, D0l:10.1007/s11630-006-0115-6.

[8] Cheong, J.-S. (2000). An analytical prediction on the pumpinduced pressure pulsation in a pressurized water reactor. Annals of Nuclear Energy, vol. 27, no. 15, p. 1373-1383, D0l:10.1016/S0306-4549(99)00132-2.

[9] Wang, C., Yi, T.,Wu, Z., Liu, H., Liang, J. (2009). Analysis on pressure fluctuations of unsteady flow field in mixed-flow main coolant pump. Journal of Power Engineering, vol. 29, no. 11, p. 1036-1040.

[10] Bing, H., Cao, S., Tan, L., Zhu, B. (2013). Effects of meridional flow passage shape on hydraulic performance of mixed-flow pump impellers. Chinese Journal of Mechanical Engineering, vol. 26, no. 3, p. 469-475, D0l:10.3901/cjme.2013.03.469.

[11] Chan, A.M.C., Kawaji, M., Nakamura, H., Kukita, Y. (1999). Experimental study of two-phase pump performance using a full size nuclear reactor pump. Nuclear Engineering and Design, vol. 193, no. 1-2, p. 159-172, D0l:10.1016/S00295493(99)00150-8.

[12] Xie, R., Shen, F., Wang, X., Shan, Y. (2011). A new CFDbased method study on modelling design of nuclear main pump impeller. Proceedings of the Asia-Pacific Power and Energy Engineering Conference, p. 1-4, D0l:10.1109/ appeec.2011.5748945.

[13] Zhang, N., Yang, M., Gao, B., Li, Z., Ni, D., (2015). Experimental investigation on unsteady pressure pulsation in a centrifugal pump with special slope volute. Journal of Fluids Engineering, vol. 137, no. 6, p. 061103, Dol:10.1115/1.4029574.

[14] Knierim, C., Baumgarten, S., Fritz, J., Coon, M.T. (2005). Design process for an advanced reactor coolant pump for a 1400 MW nuclear power plant. ASME Fluids Engineering Division Summer Meeting and Exhibition, p. 1-7, Dol:10.1115/ FEDSM2005-77340.

[15] Kato, C., Mukai, H., Manabe, A. (2003). Large-Eddy simulation of unsteady flow in a mixed-flow pump. International Journal of Rotating Machinery, vol. 9, no. 5, p. 345-351, D0l:10.1155/ S1023621X03000320.

[16] Kato, C., Mukai, H., Manabe, A. (2002). LES of internal flows in a mixed-flow pump with performance instability. Proceedings 
of ASME Joint U.S.-European Fluids Engineering Division Conference, vol. 2, p. 1-8, D0l:10.1115/FEDSM2002-31205.

[17] Posa, A., Lippolis, A., Verzicco, R., Balaras, E. (2011). Largeeddy simulations in mixed-flow pumps using an immersedboundary method. Computers \& Fluids, vol. 47, no. 1, p. 3343, D0I:10.1016/j.compfluid.2011.02.004.

[18] Inoue, Y., Nagahara, T. (2012). Application of PIV for the flow field measurement in a mixed-flow pump. IOP Conference Series: Earth and Environmental Science, vol. 15, no. 2, p. 022011, D0I:10.1088/1755-1315/15/2/022011.

[19] Fernández, J., Blanco, E., Santolaria, C., Scanlon, T.J., Stickland, M.T. (2002). A numerical analysis of a mixed flow pump. ASME Joint U.S.-European Fluids Engineering Division Summer Conference, p. p. 791-798, D0l:10.1115/ FEDSM2002-31185.

[20] Goto, A., Takemura, T., Zangeneh, M., (1996). Suppression of secondary flows in a mixed-flow pump impeller by application of three-dimensional inverse design method: part 1-design and numerical validation. Journal of Turbomachinery, vol. 118, no. 3, p. 536-543, Dol:10.1115/1.2836701.

[21] Srivastava, S., Roy, A.K., Kumar, K. (2014). Design of a mixed flow pump impeller blade and its validation using stress analysis. Procedia Materials Science, vol 6, p. 417-424, DOI:10.1016/j.mspro.2014.07.053.

[22] Takayama, Y., Watanabe, H. (2009). Multi-objective design optimization of a mixed-flow pump. Proceedings of the ASME Fluids Engineering Division Summer Meeting, vol. 1, p. 1-9, DOI:10.1115/FEDSM2009-78348.

[23] Voke, P.R. (1996). Subgrid-scale modelling at low mesh Reynolds number. Theoretical and Computational Fluid Dynamics, vol. 8, no. 2, p. 131-143, Dol:10.1007/ BF00312367.

[24] Yang, Z., Wang, F., Zhou, P. (2012). Evaluation of subgridscale models in large-eddy simulations of turbulent flow in a centrifugal pump impeller. Chinese Journal of Mechanical Engineering, vol. 25, no. 5, p. 911-918, D0l:10.3901/ CJME.2012.05.911.

[25] Yamanishi, N., Fukao, S., Qiao, X., Kato, C., Tsujimoto, Y., (2006). LES simulation of backflow vortex structure at the inlet of an inducer. Journal of Fluids Engineering, vol. 129, no. 5, p. 587-594, D0l:10.1115/1.2717613.

[26] Piomelli, U. (1999). Large-eddy simulation: achievements and challenges. Progress in Aerospace Sciences, vol. 35, no. 4, p. 335-362, D0I:10.1016/S0376-0421(98)00014-1.

[27] Eastwood, S.J., Tucker, P.G., Xia, H., Klostermeier, C. (2009). Developing large eddy simulation for turbomachinery applications. Philosophical transactions. Series A, Mathematical, Physical, and Engineering Sciences, vol. 367, no. 1899, p. 2999-3013, D0l:10.1098/rsta.2008.0281.

[28] Ducros, F., Ferrand, V., Nicoud, F., Weber, C., Darracq, D., Gacherieu, C., Poinsot, T., (1999). Large-Eddy simulation of the shock/turbulence interaction. Journal of Computational Physics, vol. 152, no. 2, p. 517-549, Dol:10.1006/ jcph.1999.6238.

[29] Kato, C., Kaiho, M., Manabe, A. (2003). An overset finiteelement large-eddy simulation method with applications to turbomachinery and aeroacoustics. Journal of Applied Mechanics, vol. 70, no. 1, p. 32-43, D0l:10.1115/1.1530637.

[30] Posa, A., Lippolis, A., Balaras, E. (2015). Large-Eddy simulation of a mixed-flow pump at off-design conditions. Journal of Fluids Engineering, vol. 137, no. 10, p. 101302, DOl:10.1115/1.4030489.

[31] Byskov, R.K., Jacobsen, C.B., Pedersen, N., (2003). Flow in a Centrifugal Pump Impeller at Design and Off-Design ConditionsPart II: Large Eddy Simulations. Journal of Fluids Engineering, vol. 125, no. 1, p. 73-83, D0I:10.1115/1.1524586.

[32] Zhang, N., Yang, M., Gao, B., Li, Z., Ni, D. (2016). Investigation of rotor-stator interaction and flow unsteadiness in a low specific speed centrifugal pump. Strojniški vestnik - Journal of Mechanical Engineering, vol. 62, no. 1, p. 21-31, DOl:10.5545/sv-jme.2015.2859.

[33] Sang-hyuk, Lee, S.H., Wang, Y.-Q., Song, J.-L., (2010). Fourier and wavelet transformations application to fault detection of induction motor with stator current. Journal of Central South University of Technology, vol. 17, no. 1, p. 93-101, DOI:10.1007/s11771-010-0016-4. 